\title{
Chronometric evidence for entrained attention
}

\author{
TIM MARTIN, ROBERT EGLY, JON M. HOUCK, JOEL PEARSON BISH, \\ BRANDON D. BARRERA, DUSTIN C. LEE, and CLAUDIA D. TESCHE \\ University of New Mexico, Albuquerque, New Mexico
}

\begin{abstract}
Recent theories of dynamic attention have renewed the interest in temporal context as a determinant of attention. The mechanism of dynamic attention remains unclear, and both stochastic time perception processes and deterministic oscillators are possible. The results of Experiment 1 demonstrate that attention can be guided by isochronous series of warning stimuli and that elapsed time cannot fully account for this effect. Experiment 2 indicates that temporal structure can be used over a limited range of time. The results of Experiment 3 indicate that temporal pattern, rather than variability, is a determinant of temporally focused attention. The results of Experiment 4 demonstrate that a coupled oscillator is a better predictor of reaction time than a stochastic timing mechanism is, under certain assumptions.
\end{abstract}

The concept of attention is invoked to explain limitations on the amount of information that cognitive systems can process per unit time and differences in performance to identical input under different antecedent conditions. Although there are many approaches to explaining such observations, it seems appropriate to summarize attention as a process modulating the selection, sensitivity, and efficiency of a limited capacity for information processing (cf. Kinchla, 1992; Posner, Snyder, \& Davidson, 1980). Such a concept implies a dynamic change, and indeed, many dynamic phenomena related to attention have been described. We investigated the ability of deterministic oscillator and stochastic timing models to account for the temporal allocation of attention, using both qualitative and quantitative approaches to chronometric data.

A theoretical account of dynamic attention by Jones (1976) has been the focus of several recent studies (Barnes \& Jones, 2000; Large \& Jones, 1999; McAuley \& Kidd, 1998; Olsen \& Chun, 2001). According to dynamic attending theory (DAT; Jones, 1976; Jones \& Boltz, 1989; Large \& Jones, 1999), attention is guided through time by internal oscillators termed attending rhythms. Attending rhythms are coupled to the environment through perception. This coupling causes them to become correlated with the environment through a process of entrainment when the temporal structure of the environment is autocorrelated. Attention is then focused on an epoch with an intensity that depends on the phase relationship (i.e., the degree of correlation) between the environmental event stream and the attending rhythms. Specifically, the greater the degree of synchronization between the internal oscillator and the external sequence, the narrower and more intense the attentional pulse becomes.

The authors thank Harold Delaney for statistical advice and Timothy Goldsmith for helpful comments on an earlier draft of this article. Correspondence concerning this article should be addressed to T. Martin, Logan Hall, University of New Mexico, Albuquerque, NM 87131 (email: tma2010@yahoo.com).
Much of the evidence for DAT comes from nearthreshold detection tasks for timing information. Jones and Yee (1997) found that temporal just-noticeable differences were smaller for intervals embedded in rhythmic sequences than for randomly timed sequences, even though the rhythmic sequences were more variable. Jones and Boltz (1989) found that discrimination between the duration of two melodies was influenced by whether or not the last note of one melody violated a temporal pattern established by the preceding notes. Large and Jones (1999) found that time change detection accuracy was better when stimuli occurred at unexpected times and that duration estimation accuracy was better when stimulus events occurred at expected times, where expected times were defined by DAT. Barnes and Jones (2000) found that duration judgments about a standard and a comparison interval were most accurate when the temporal context preceding the standard had a simple harmonic relationship to the standard interval. In all of these studies, temporal context altered sensitivity to temporal information in a direction consistent with a coupled oscillator mechanism.

The use of near-threshold detection measures of timing has invited comparisons between DAT and temporal information processing theories, such as the multiplelook model (Drake \& Botte, 1993), the average Weber model (Monahan \& Hirsh, 1990), and Povel's clock model (Povel, 1981; Povel \& Essens, 1985). These models assert the distribution of a psychological duration code extracted from environmental durations, which is further asserted to relate to performance on various time perception tasks.

\section{Chronometric Measures of Attention}

Because of the ambiguity involved in the interpretation of performance benefits, near-threshold detection performance may not be optimal for the testing of theories of attention (Posner, 1980; Posner et al., 1980). Nearthreshold response biases are difficult to rule out as explanations. Chronometric measures of performance for targets well above threshold provide another approach to 
studying attention and minimize the contribution of response biases. Although some have proposed that any information about a target acts to facilitate reaction time (RT) by lowering a response criterion (Duncan, 1980, 1984), this hypothesis has not received wide support (Garner, 1985; Garner, 1987; Tsal, 1983). Some types of information, such as spatial cues about likely location, improve performance speed without appreciably degrading accuracy. Without a speed-accuracy tradeoff for RT (or a constant $d^{\prime}$ for detection), it is difficult to argue that response biases have been altered by the cue. Simple RT (SRT) is not optimal, because it is impossible to rule out the effects of cuing on motor preparation. Therefore, choice RTs (CRTs), where the choice is made along a dimension other than the cued dimension, are considered better reflections of attention. Cues that direct attention should improve the sensitivity and efficiency of information processing at attended points.

In order to use CRT as a measure of attention related to temporal structure, a good starting point is one with the simplest temporal structure, an isochronous sequence. Such a sequence establishes an easily perceptible temporal context. Targets embedded in such a sequence provide a way of sampling the level of attention being allocated at the time of target onset. For example, suppose an isochronous series of visual warning stimuli (WSs) is presented to observers with some period, followed by a target one period after the last WS, requiring a choice reaction based on color. The series would serve as a temporal cue. According to DAT, the series of WSs should afford dynamic attending. The attending rhythms should become entrained to the series, an attentional pulse should reach a maximum one period after the last WS, and a target occurring one period after the last WS should be processed more quickly than an uncued target or a target cued by only a single WS. There should not be a speed-accuracy tradeoff, because the gain in speed is predicted to be a function of increasing processing efficiency, not a change in response bias.

The same prediction cannot be made from the models of temporal information processing discussed here, at least in their current form. These theories do not specify a relationship between the time code and attention. They give no reason to suspect that information about target onset time will have consequences for the processing of information about nontemporal dimensions, such as color. A response bias account of a benefit to CRT is possible, but this would entail a speed-accuracy tradeoff, as was discussed above.

Nevertheless, if one asserts that a time code can guide attention, predictions about CRT to an uncued dimension can be made from timing theories. Something of the kind would seem necessary to explain the effects of the symbolic cuing of intervals (Kingstone, 1992). The asserted relationship between attention and the time code can take many forms, an issue that we will discuss in detail in Experiment 4. For the present, it will suffice to assume that information about when a stimulus is likely to occur can be used to focus attention at that most likely time. Then the intervals in a WS series could be encoded and used to allocate attention to the points in time defined by the statistical moments of the intervals. This too would produce a shorter CRT than that to targets following an isochronous series of WSs.

\section{The WS Paradigm and the Conditional Probability Explanation}

In addition to coupled oscillator and stochastic timing accounts of a benefit to CRT following an isochronous sequence, theoretical explanations of performance in the WS paradigm provide another explanation for such a benefit that depends only on elapsed time. Some work in the WS paradigm also indicates that the predicted benefit may not occur. In the WS paradigm, a WS is first presented to bring participants to a state of readiness to respond. This is followed by the target stimulus at some lag usually referred to as the preparatory interval, or foreperiod. The duration of the foreperiod from onset of the WS to onset of the target is generally referred to as the interonset interval, or IOI. The target stimulus requires some response, such as a detection, a speeded simple reaction, or a choice reaction (for reviews, see Niemi \& Näätänen, 1981, and Teichner, 1954).

When foreperiods are presented in homogenous blocks, RT increases with increasing IOI (Klemmer, 1956). If foreperiods are randomly mixed within blocks, this relationship is reversed, so that RT decreases as IOI increases (Woodrow, 1914). In both of these cases, the ability of the observer to estimate the foreperiod is typically considered an important causal variable (Requin \& Granjon, 1969). Performance on time interval estimation tasks is known to be more precise for shorter intervals than for longer across a wide range of durations (Allan, 1979; Poppel, 1997; Woodrow, 1930). Thus, in the case of blocked presentation, when observers know that each trial will have the same IOI, it is primarily their ability to use this information to focus attention at a particular point in time that limits performance.

In the case of mixed IOIs, however, another factor influences the allocation of attention. If the probability distribution of IOIs is uniform, on any given trial, the conditional probability of a target, given that no target has yet occurred, increases with time. Therefore, participants may increase their allocation of attention over the trial interval until a target occurs according to the subjective probability of target onset. This allocation of attention with subjective probability conceals the effect of time estimation (Näätänen, 1970; Requin \& Granjon, 1969).

Now consider our proposal to measure CRT to targets following isochronous sequences of WSs. If the number of WSs is varied within subjects, the time elapsed since the first WS increases with the number of WSs, as does the conditional probability of a target if the frequency distribution of the number of WSs is uniform. Therefore, any observed benefit may be due to an increasing allocation of attention on the basis of the conditional proba- 
bility, and not to any entrainment mechanism. Note that this timing explanation, in contrast to one that asserts encoding of intervals, does not rely on the rhythmic nature of the sequence at all, but only on the elapsed time.

One way of controlling for elapsed time is to present trials of the same duration with and without additional isochronous series of WSs following the first WS. ${ }^{1}$ Although this manipulation reduces the effective foreperiod in trials with isochronous WSs to the rate-defining IOI and, thus, time estimation of the last interval should be better for these trials, this is relevant only for a blocked design. Recall that in blocked designs, the shortest IOI causes the shortest RT, whereas in mixed designs, the longest IOI causes the shortest RT. In either case, trials with a single WS and a foreperiod equal to the rate-defining IOI can serve as a control to eliminate the possibility that it is merely time estimation of a shorter interval that causes any benefit.

Evidence from the WS paradigm suggests that no benefit may occur to CRT following an isochronous series of WSs. To disentangle the differential effects of time estimation and conditional probability described above in the WS paradigm, experimenters introduced an isochronous series of time-marking stimuli between the WS and the target to reduce the influence of time estimation on RT (Granjon, Requin, Durup, \& Reynard, 1973; Requin $\&$ Granjon, 1969). This procedure had different effects, depending on whether IOIs were blocked or mixed. For blocked IOIs, where time estimation was considered the major influence on RT, time markers did indeed reduce RT for longer IOIs (Simon \& Slaviero, 1975). However, Niemi and Näätänen (1981) noted that the isochronous series did not simply relieve participants of the need to estimate a temporal interval, but also reduced the effective foreperiod to the interval between the last time marker and the target. Thus, the benefit could be explained as simply another instance of the short IOI = short RT relationship already noted for blocked designs.

In the case of mixed designs, Requin and Granjon (1969) found that the decrease in simple RT with increasing IOI was enhanced by the time markers (i.e., the slope of the function became more negative relative to conditions without time markers) over the range of IOIs from 8 to $28 \mathrm{sec}$, just as would be predicted if the time interval estimation ability of participants and the conditional probability of a target with elapsed time were working in opposition. In addition, however, they found an overall cost of time markers. Even though RT with time markers decreased at a greater rate with increasing IOI, it was longer overall than RT without time markers.

These results would seem to contradict the prediction that isochronous sequences should enhance performance by providing information about time. However, the experimental designs of Requin and Granjon (1969) and Granjon et al. (1973), although quite adequate for the hypotheses they were testing, are inadequate to assess the phenomenon of temporally guided attention in the presence of temporal structure. First, their own interpretation of the cost to performance with time markers was that the introduction of time markers with mixed IOIs changed the nature of the task from simple reaction to a go-no-go task. This is because the time markers occur at times when a target could otherwise appear, so the observer must decide at each stimulus onset whether or not the stimulus is a target. This is a form of choice reaction, and choice reactions are known to be slower than simple reactions (Luce, 1984, p. 208). Also, Requin and Granjon (1969) and Granjon et al. used relatively long IOIs, on the order of several seconds. It has been suggested that temporal information processing in the milliseconds range and the seconds range may be mediated by different systems (Getty, 1975; Poppel, 1997). This would suggest that the results of Requin and Granjon (1969) and of Granjon et al. may be limited to intervals on the order of seconds, whereas attention may be directed to points in time only by temporal structures in the millisecond range. This would be similar to the situation with synchronized tapping, where humans are able to effectively synchronize their response to the onset of a stimulus over a limited (approximately $0.6-$ to $5-\mathrm{Hz}$ ) range of rates (Fraisse, 1982).

\section{The Present Experiments}

In Experiment 1, we sought to address the issue of whether time markers cause a benefit for CRT when response factors and elapsed time are adequately controlled. Observers were presented with a single WS, followed at a variable IOI by a target requiring a choice reaction. On some trials, the IOI was filled with additional WSs. If these additional stimuli caused a benefit, we would also address the issue of whether it could be explained by the increased allocation of attention with the increasing conditional probability of a target on a given trial, or if an attention process that is guided by time markers is necessary to explain it. In Experiment 2, we explored the notion that one reason previous studies may have failed to show the predicted benefit may be the time scale defined by the IOIs and the rates of time marker presentation used in those earlier experiments. In Experiment 3, our goal was to develop a more direct comparison between stochastic and oscillatory models of temporally guided attention by independently manipulating variability and temporal pattern. Finally, in a concluding experiment, we took a more formal approach, computing a theoretical value of attention for individual trials and observers from formal models that link attention and stimulus onset times via either coupled oscillator or stochastic timing mechanisms, allowing us to estimate the ability of such models to independently account for CRT data.

We elected not to use catch trials in the first three experiments. The purpose of catch trials is to control the probability of target onset, and their use is particularly important when simple RT is used as a measure, since without catch trials simple reactions at the longest IOI reduce to a single alternative (Luce, 1984, p. 55). However, in the context of the present experiment, the choice 
reaction requires that the participants attend to a target occurring even at the longest foreperiod, and the absence of catch trials gives a special meaning to the longest foreperiod condition: The target will occur with a probability of 1 . Since the probability of target onset is maximum without catch trials, any benefit seen in the longest IOI condition without additional WSs is the maximum possible benefit conferred by the probability of target onset.

\section{EXPERIMENT 1}

\section{Hypotheses}

The conditional probability hypothesis. If the benefit to RT over time with time markers in mixed IOI experiments is due only to the increasing allocation of attention with an increasing conditional probability of a target, there should be no difference between trials with and without time markers that have the same elapsed time since the onset of the first WS, but there should be an overall decrease in RT over elapsed time. This prediction follows from the fact that trials with and without additional WSs have the same elapsed time and the identical conditional probability of a target occurrence at each time point.

The temporal structure hypothesis. If the benefit to RT is due to a process whereby attention and the temporal structure of the environment become increasingly correlated, whether through a coupled oscillator or a temporal information processing mechanism, there should be a greater reduction in RT on trials with time markers than that observed on trials with the same elapsed time but no time markers.
The cost hypothesis. If the cost of time-marking stimuli found in Requin and Granjon (1969) is not partially accounted for by the confound between simple and choice reactions or the slow rate of time marker presentation used, CRT should be slower in trials with additional warning stimuli.

\section{Method}

Participants. Ten undergraduate psychology students ( 7 females, 1 left-handed) at a large research university participated for extra credit in a methodology class. Ages ranged from 20 to 38 years, with a mean of 24 years. All reported normal or corrected vision.

Stimuli and Apparatus. The stimuli were white, red, and green circles (capital letter "O" in Superlab 1.03 system font) presented centrally, with an intensity well above threshold and subtending approximately $1.5^{\circ}$ of visual angle. Stimuli were presented on a Dell 1025 HTX monitor with 16-bit color, $1,024 \times 768$ pixel spatial resolution, and a refresh rate of $60 \mathrm{~Hz}$, controlled by a Dell Dimension XP5 PC. All the stimuli were presented for a duration of $50 \mathrm{msec}$. Responses were collected from a Microsoft Intellimouse 1.1A.

Design. Figure 1 illustrates each condition in the experiment. The design is a 2 (empty interval vs. time marker) $\times 3$ (elapsed time) repeated measure factorial, with two additional hanging cells that serve as control conditions: a condition in which the target occurs after a random intertrial interval (ITI) without warning, which we term the uncued CRT condition, and a condition in which a single WS is followed by the target at an IOI equal to the period of time marker presentation, which we refer to as the shortest IOI condition. In the empty interval conditions, a WS is followed at a variable IOI by a target. In time marker conditions, the first WS is followed by additional WSs presented at a constant rate.

Procedure. The participants were informed of the experimental procedure and of their rights as participants, and informed consent was obtained. Demographic information was obtained, including age, sex, vision, and estimated years of music training.

The participants were told that they would see red and green circles presented in the context of white circles and that, in all cases,

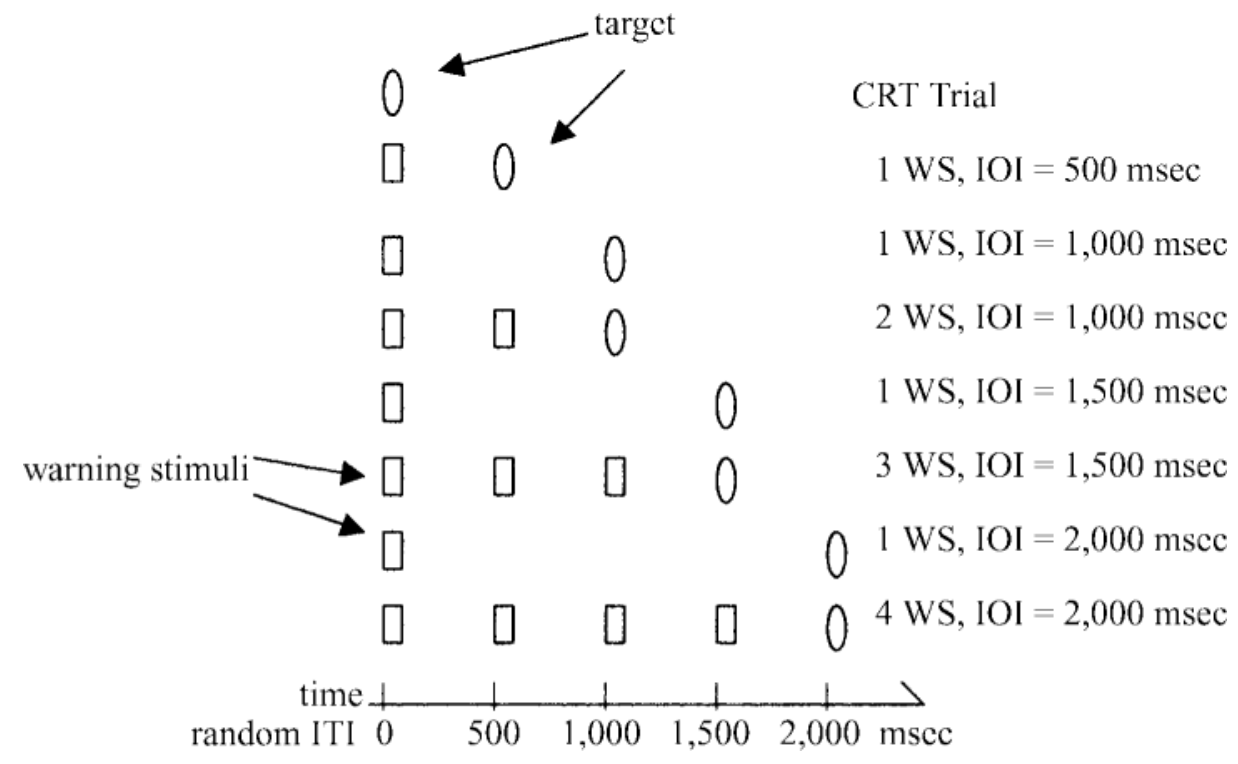

Figure 1. Illustration of the experimental conditions in Experiment 1. Times are for Experiment 1. Experiment 2 used the same design, but the interonset intervals (IOIs) were 4, 8, 12, and 16 sec, with time markers presented every 4 sec. CRT, choice reaction time; WS, warning stimulus; ITI, intertrial interval. 
their task was to press the left button if they saw a green circle and the right button if they saw a red circle. Both speed and accuracy were emphasized. The participants were told that the white circles would act as warnings that targets would occur soon, and they were told that they could see between zero and four such WSs. They were given no other information about the meaning of the different conditions.

The participants were seated at a desk facing the computer. No constraints were placed on head or eye position. All the trials were presented in a different random order for each participant and were separated by an ITI that varied from 3 to $4 \mathrm{sec}$ in uniformly distributed $100-\mathrm{msec}$ increments. On empty interval and time marker trials, the IOI separating the first WS from the target was varied from 500 to $2,000 \mathrm{msec}$ in $500-\mathrm{msec}$ increments. On time marker trials, an additional set of one, two, or three stimuli followed the first WS at constant onset-to-onset intervals of $500 \mathrm{msec}$. Each participant was observed in each condition 24 times, for a total of 192 trials. Each experimental session lasted approximately $25 \mathrm{~min}$.

\section{Results}

After error trials were removed, a grand mean and a standard deviation were calculated for each participant. Any responses that fell outside three standard deviations of the mean for that participant were removed. This resulted in the loss of less than $5 \%$ of the data in all cases. Mean RTs for each participant and condition were then calculated. The means for each condition are presented in Figure 2. A pseudo-omnibus ${ }^{2} 3$ (elapsed time) $\times 2$ (time markers) repeated measures analysis of variance (ANOVA) revealed a main effect of elapsed time [Hotelling's trace $F(2,8)=9.131, p=.009]$. The observers were quicker to respond as elapsed time increased. There was also a main effect of time markers $[F(1,9)=28.903, p<$ $.0005]$. The observers were faster with time markers than without. There was no detectable interaction $[F(2,8)=$ $0.838]$.

Multivariate repeated measures ANOVAs served as planned comparisons, with Bonferroni-adjusted $\alpha=$
Table 1

Mean Differences for Comparisons in Experiment 1

\begin{tabular}{lcccc}
\hline & Mean Difference & \multicolumn{1}{c}{$S E$} & U95\%CI & L95\%CI \\
\hline Elapsed time & -5.503 & 9.737 & 16.529 & -27.535 \\
& -8.533 & 9.985 & 14.06 & -31.126 \\
& -8.512 & 9.494 & 12.97 & -29.994 \\
Time markers & -22.728 & 9.765 & -0.632 & -44.824 \\
& -20.961 & 11.119 & 4.198 & -46.12 \\
& -17.638 & 10.957 & 7.155 & -42.431 \\
IOI $=1 \mathrm{sec}$ & 17.226 & 8.641 & -10.857 & 45.309 \\
IOI $=1.5 \mathrm{sec}$ & 29.653 & 7.786 & 4.349 & 54.956 \\
IOI $=2 \mathrm{sec}$ & 38.781 & 11.784 & 0.487 & 77.075 \\
\hline
\end{tabular}

Note-Multivariate repeated measures utilize matrices of difference scores. With three or more levels, there is more than one set of difference scores that yield identical results, subject to the constraint that they be orthogonal. The multivariate $F$ ratio then tests the experimental hypothesis that at least one of the mean difference scores is different from zero. $S E$, standard error of the mean; U95\%CI, upper $95 \%$ confidence interval; L95\%CI, lower 95\% confidence interval; IOI, interonset interval.

$.05 / 5=.01$. Mean difference scores and confidence intervals for each analysis are presented in Table 1. Note that because we are using a multivariate analysis (see Maxwell \& Delaney, 2003, pp. 654-658), there are three difference scores associated with each of the first two tests, corresponding to a set of orthogonal difference scores among four levels. The first comparison tested the effect of elapsed time without time markers and, so, included empty interval trials with one WS and IOIs of $500,1,000,1,500$, and $2,000 \mathrm{msec}$. This test revealed no main effect of elapsed time [Hotelling's trace $F(3,7)=$ $4.342, p=.05]$. The second comparison tested the effect of time markers and, so, included one-, two-, three-, and four-WS trials. Note that the IOIs in this test are the same as those included in the first comparison and that the first mean is the same. This test revealed a significant

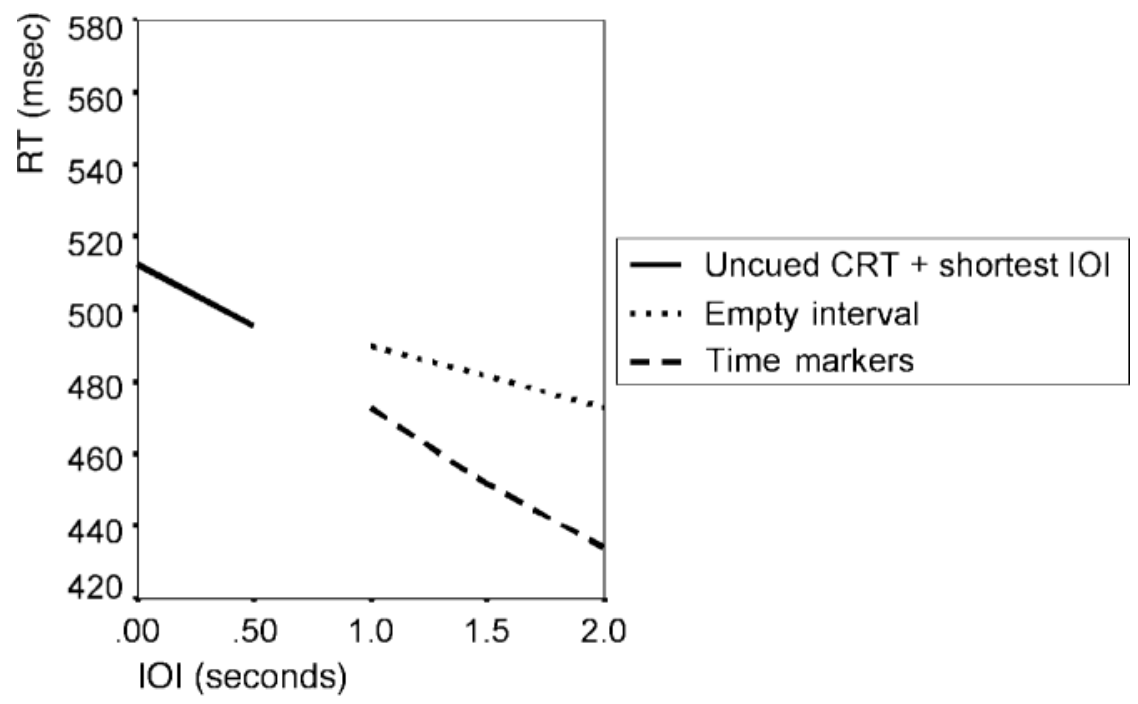

Figure 2. Mean reaction time (RT) for each condition in Experiment 1. The solid line indicates uncued trials and shortest interonset interval (IOI) trials, the dotted line indicates empty interval trials, and the dashed line indicates time marker trials. CRT, choice RT. 
effect [Hotelling's trace $F(3,7)=9.929, p=.006$ ], such that the observers were faster with an increasing number of time markers. Three more tests compared CRT following time markers with CRT following empty intervals at each IOI. With an IOI of $1,000 \mathrm{msec}$, there was no detectable effect of time markers [ $t(9)=1.993, p=.077]$, although the trend was in the direction of faster reactions with time markers. At a 1,500-msec IOI, time markers significantly reduced CRT $[t(9)=3.808, p=.004]$. At a 2,000-msec IOI, time markers significantly reduced CRT $[t(9)=3.291, p=.009]$.

We analyzed errors for indications of a speed-accuracy tradeoff in two ways. First, the proportion of correct responses in each condition was calculated and analyzed exactly as CRT was. This analysis revealed no main effects or interactions. Second, response category (correct or error) was entered as a categorical dependent variable into a logistic regression, with trial type as a categorical predictor, on an individual observer level. This series of regressions failed to predict response category at a greater than chance level for any observer.

Finally, musical training was recorded in each experiment reported here, including this experiment, by asking the participants to estimate the number of years that they had actively played a musical instrument, sung, or danced in a formal setting (class, a dance troupe, etc.). This variable was analyzed both as a continuous covariate and as a dichotomous covariate (some or none). It was not a significant predictor of CRT or a significant covariate in any analysis and was, therefore, not included in the analyses reported in this or the subsequent experiments.

\section{Discussion}

The temporal structure hypothesis predicted a benefit to CRT with time markers in a mixed IOI WS paradigm, in addition to the benefit conferred by the simple passage of time. The results of this experiment supported that prediction, clearly showing an additional benefit of time markers, relative to trials with the same elapsed time and conditional probability of target onset. This effect cannot be due to a shorter effective IOI between the last WS in a series and the target, because trials with a single WS and the same IOI (500 msec) obtained longer CRTs, as would be expected in a mixed IOI design.

One possible alternative explanation for the benefit observed here is that time markers afforded a counting strategy that allowed the participants to better estimate the passage of time and, thus, allocate attention on the basis of the increasing subjective probability of target onset, rather than allocating attention by using the isochronous temporal structure of the time marker series. For sufficiently long temporal intervals, observers are known to initiate an internal cyclical process to subdivide the interval into subintervals and, then, count the subintervals to judge the passage of time (Killeen \& Weiss, 1987). Time markers filling an interval may allow participants to subdivide on the basis of the intervals defined by the time markers, rather than by an internal cyclical process, and simply count the time markers. Grondin,
Meilleur-Wells, and Lachance (1999) found that voluntary counting did not significantly improve timing performance for intervals under about $1 \mathrm{sec}$ but that, for longer intervals, counting improved interval discrimination performance. We failed to detect an effect of time markers at an IOI of $1 \mathrm{sec}$ but did detect a benefit for longer IOIs, a result that is consistent with Grondin et al.'s estimate. This issue is addressed in Experiment 3.

There was no evidence of a speed-accuracy tradeoff. Therefore, it would be difficult for timing theories to explain the present results as a simple lowering of a response criterion at the time of target onset caused by the temporal information. Rather, it seems that attention becomes focused at times defined by the WS sequence, and this facilitates CRT with no detectable cost.

In Experiment 2, we extended the design of Experiment 1 to a much longer time scale, in order to assess another possible source of the cost of time markers found by Requin and Granjon (1969).

\section{EXPERIMENT 2}

Requin and Granjon (1969) found that time markers resulted in a cost to performance, rather than a benefit, but this comparison was between simple reaction and go-no-go performance. The results from Experiment 1 clearly demonstrated that when response (simple vs. choice) was adequately controlled, an isochronous series of WSs caused a benefit to CRT.

Another possible explanation for the difference between the present results and those of earlier WS paradigm work (Granjon et al., 1973; Requin \& Granjon, 1969 ) is the difference in presentation rate for time markers. It is well established that behavioral entrainment has upper and lower limits (Fraisse, 1982). The upper limit appears to reflect mechanical limitations on effectors, which simply cannot move effectively more quickly than about $5 \mathrm{~Hz}$. The lower limit, on the other hand, would appear to reflect cognitive limitations, since there is no physical reason that a finger or a hand could not move in synchrony with stimulus onsets occurring every $1.8 \mathrm{sec}$ or less.

Mechanisms that limit behavioral entrainment may also affect attentional allocation for increasingly lengthy interval durations. We explored this possibility in Experiment 2 by repeating the pattern of stimulus presentation and relative temporal relationships used in Experiment 1, but modifying the frequency of WS presentation to $0.25 \mathrm{~Hz}$ to encompass durations comparable to those used in Requin and Granjon (1969) and Granjon et al. (1973). Since one stimulus was presented every $4 \mathrm{sec}$ in time marker conditions, IOIs were altered accordingly, so that the shortest IOI was $4,000 \mathrm{msec}$ and the longest was $16,000 \mathrm{msec}$, and ITIs were lengthened to a range of between 12 and $14 \mathrm{sec}$, uniformly randomly varied in 100-msec increments.

\section{Hypotheses}

The arbitrary range hypothesis. If temporal structure over an arbitrary range (here, sampled only up to $0.25 \mathrm{~Hz}$ ) affords attentional allocation to time, the pattern of 


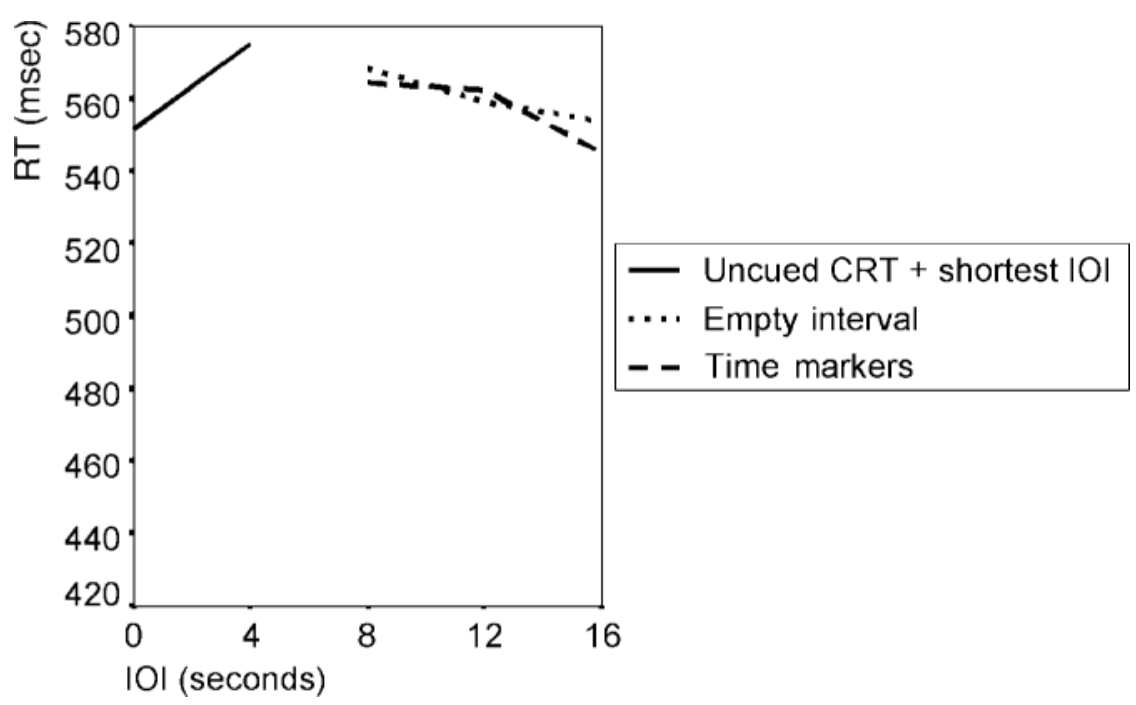

Figure 3. Mean reaction time (RT) for each condition in Experiment 2. The solid line indicates uncued trials and the shortest interonset interval (IOI) trials, the dotted line indicates empty interval trials, and the dashed line indicates time marker trials. CRT, choice RT.

means should be similar to that obtained in Experiment 1 , with shorter CRTs after time markers than after trials with only a single WS.

The limited range hypothesis. If accurate temporal allocation of attention is possible only over a limited and relatively fast range of WS frequencies near that used in Experiment $1(2.5 \mathrm{~Hz})$, there should be no effect of time markers in the present experiment.

\section{Method}

Participants. Eleven undergraduate psychology students and 1 graduate psychology student ( 7 females, 1 left-handed) at a large research university participated for extra credit in a methodology class (the graduate student participated voluntarily, without compensation). Ages ranged from 18 to 35 years, with a mean of 24 years. All reported normal or corrected vision.

Apparatus. All the stimuli and equipment were identical to those in Experiment 1.

Design. The design of Experiment 2 was identical to that in Experiment 1.

Table 2

Mean Differences for Comparisons in Experiment 2

\begin{tabular}{lcccc}
\hline & Mean Difference & $S E$ & U95\%CI & L95\%CI \\
\hline Elapsed time & -6.846 & 13.42 & 22.402 & -36.094 \\
& -9.193 & 15.215 & 23.967 & -42.353 \\
& -5.423 & 15.605 & 28.587 & -39.433 \\
Time markers & -10.78 & 15.629 & 23.283 & -44.843 \\
& -1.962 & 18.174 & 37.647 & -41.571 \\
& -17.357 & 9.104 & 2.485 & -37.199 \\
IOI $=1 \mathrm{sec}$ & 3.936 & 8.821 & -23.46 & 31.332 \\
IOI $=1.5 \mathrm{sec}$ & -3.296 & 8.8 & -30.627 & 24.04 \\
IOI $=2 \mathrm{sec}$ & 8.638 & 8.761 & -18.572 & 35.849 \\
\hline
\end{tabular}

Note-SE, standard error of the mean; U95\%CI, upper 95\% confidence interval; L95\% CI, lower 95\% confidence interval; IOI, interonset interval.
Procedure. All the procedures were identical to those in Experiment 1 , with the exception of the following changes to the timing of stimuli. The ITI was lengthened so that it would be long relative to the rate of stimulus presentation within trials. The ITI ranged between 12 and $14 \mathrm{sec}$, randomly varied according to a uniform distribution of $100-\mathrm{msec}$ increments. The IOI separating the first WS from the target was varied from 4,000 to $16,000 \mathrm{msec}$ in 4,000 msec increments. On half of the WS trials with IOIs of 8,000 , 12,000 , or $16,000 \mathrm{msec}$, an additional set of one, two, or three stimuli followed the first WS at constant onset-to-onset intervals of $4,000 \mathrm{msec}$. Again, no catch trials were used, in order to maximize the probability that a target would occur at the longest IOI. In addition, the participants saw trials in which no WSs preceded the target, which we refer to as uncued CRT trials. Each participant was observed in each condition 24 times, for a total of 192 trials. An experimental session lasted approximately $80 \mathrm{~min}$.

\section{Results}

As in Experiment 1, error trials were first removed, a grand mean and standard deviation were calculated for each participant, and any responses outside three standard deviations of that observer's mean were removed. This resulted in the loss of less than $5 \%$ of the data in all cases. Means were then analyzed with a pseudo-omnibus 3 (elapsed time) $\times 2$ (time markers) ANOVA, followed by planned comparisons (Bonferroni-adjusted $\alpha=.05 / 5=$ $.01)$.

Means for each condition are presented in Figure 3. Mean differences and confidence intervals for the comparisons are presented in Table 2. The pseudo-omnibus test revealed no main effects or interactions (all $F \mathrm{~s}<$ 1). Comparisons were performed despite this lack of significant results, because this test does not include the mean RTs for one WS at the shortest IOI.

The first comparison tested the effect of elapsed time without time markers and, so, included empty interval tri- 
als with IOIs of 4,000, 8,000, 12,000, and 16,000 msec. This test revealed no main effect of IOI [Hotelling's trace $F(3,9)=1.515, p=.276]$. The second comparison tested the effect of time markers and, so, included one-, two-, three-, and four-WS trials. This test revealed no significant effect of time markers [Hotelling's trace $F(3,9)=$ 3.777, $p=.053]$. Three more tests were run to examine the effect of time markers to elapsed time at each comparable level of IOI. There was no detectable effect of time markers at 8,000-msec IOI $[t(11)=0.446], 12,000-\mathrm{msec}$ IOI $[t(11)=-0.374]$, or $16,000-$ msec IOI $[t(11)=$ $0.986]$.

The pattern of means in Figure 3 revealed the following unanticipated result: CRTs to stimuli following WSs were generally longer than uncued CRTs. Therefore, in addition to the planned comparisons, we used a post hoc comparison to probe the difference between uncued CRT trials (i.e., targets occurring after a random ITI with no warning) and trials with one WS and an IOI of $4 \mathrm{sec}$ (i.e., the shortest IOI). This comparison did not achieve significance with post hoc correction of alpha $[t(11)=$ $-2.549, p=.023]$.

As in Experiment 1, there were no main effects or interactions of conditions on the proportion of correct responses.

Because of the relative paucity of results, a qualitative post hoc analysis was also performed for both Experiment 1 and Experiment 2. Observed power in Experiment 1 was .94, whereas in Experiment 2 it was .68. The frequency of benefit of time markers at the longest IOI was calculated for both. In Experiment 1, all 10 observers showed a benefit of time markers, relative to elapsed time. In contrast, in Experiment 2, exactly half of the observers were actually slower with time markers than without.

\section{Discussion}

The effect of time markers on CRT failed to reach statistical significance in Experiment 2. Mean CRT for twoand four-WS conditions was only 3.9-8.6 msec shorter than that in empty interval conditions with the same elapsed time, and the CRT in the 12-sec empty interval condition was actually $3.3 \mathrm{msec}$ shorter than that in the three-WS condition. The effect of elapsed time with time-marking stimuli was marginal $(p=.053)$, but since this effect included both temporal structure and conditional probability components, we cannot firmly conclude that temporal structure provided a benefit for performance. In addition, there was no detectable benefit of WSs, relative to uncued CRT.

A comparison of these results with those in Requin and Granjon (1969) reveals some similarity. They also failed to detect the benefit of elapsed time without time markers at IOIs between 8 and $28 \mathrm{sec}$. With time markers (rate, $0.25 \mathrm{~Hz}$ ), elapsed time did cause a benefit, with longer intervals obtaining shorter SRTs, but overall there was a cost to time markers, relative to empty foreperiods. In the present experiment, using CRT rather than SRT, there was no detectable cost or benefit, although the (non- significant) trend was for a benefit of elapsed time, with the trend with time markers being inconsistent.

Although this is a null result and, therefore, no firm conclusions can be drawn about the validity of the arbitrary range hypothesis, it does suggest that the effect of temporal structure at the much slower rate used here is likely considerably smaller than the temporal structure effect at the faster rates used in Experiment 1. A qualitative comparison of Experiments 1 and 2 suggests that explicit consideration of the time scale is important in developing more complete accounts of temporal attention. Temporal integration functions, whereby the influence of a stimulus onset degrades over time, might be good candidates for such an addition to current entrainment and timing models.

\section{EXPERIMENT 3}

Experiment 1 demonstrated that temporal cuing by a series of isochronous WSs causes an allocation of attention, and not merely a change in perceptual or response bias. According to DAT, the allocation of attention is the result of the entrainment of an internal oscillator to the series of WSs. Time code theories could also explain the results, given some relationship between an internal time code of the external sequence and attention. Experiments 3 and 4 were attempts to characterize more specifically the nature of the mechanism that guides attention to time in the presence of temporal structure. In the present experiment, the variability and rhythmic structure of IOIs were manipulated in an attempt to discriminate the predictions of coupled oscillator models from those of statistical timing models.

Experiment 3 takes as its starting point the results of Jones and Yee (1997). They measured the performance of observers trying to detect timing perturbations in sequences. They found greater sensitivity for sequences that were rhythmic than for what they termed irregular sequences, despite the fact that the rhythmic sequences had higher variability than the irregular ones. Their interpretation of this result was that the observers used temporal relations among elements, rather than statistical moments of temporal distributions, to alter sensitivity to time changes.

In Experiment 3, we extended the logic of Jones and Yee (1997) to the measurement of attention by CRT along an uncued dimension (color), rather than detection along a cued dimension (time). We cued the time of target onset by presenting sequences that were isochronous, rhythmic, or random, while controlling IOI and variability. If statistical moments of distributions of IOIs determine performance, one would expect an effect of variability, so that increasing variability would cause longer CRTs, but no effect of temporal structure. If, on the other hand, a set of coupled oscillators is used to track complex sequences, one would expect similar performance for targets following both isochronous and rhythmic sequences: Both CRTs should be shorter than RTs to tar- 
gets following random sequences. The key difference between these two theories is in their predictions regarding the rhythmic sequences. The temporal code for rhythmic sequences should be the same as that for random sequences with the same variance, whereas coupled oscillators with reasonably efficient coupling quickly emulate rhythmic patterns, thereby making the representation of rhythmic sequences more similar to those of isochronous sequences.

The other alternative explanation given for the results of Experiment 1 was that the time markers afforded an explicit counting strategy that enabled the participants to better judge the passage of time and, thus, enhanced their ability to allocate attention on the basis of the subjective probability of target onset, which increases with elapsed time. Killeen and Weiss (1987) derived a general framework for timing and counting that applies to a wide class of timing theories and situations. One of their general results was that when observers subdivide an empty interval by initiating a cyclical process (such as verbalizing), variability in timing depends on variability in the number of subintervals generated by the cyclical process and variability in the duration of the subintervals themselves. In Experiment 3, the number of subintervals is fixed by the time markers, so only variability in the duration of subintervals defined by the time markers should affect the distribution of the time code. In this case, an explicit counting strategy would lead to the same pattern of results as that predicted by time estimation alone: Participants should respond most quickly to targets following isochronous sequences and should respond more slowly after sequences with increasing variability regardless of temporal pattern.

\section{Hypotheses}

Statistical moment code and counting hypotheses. If a time code based on statistical moments of interval distributions is used to allocate attention, CRT should be an increasing function of variability, but the exact temporal structure should not matter. Likewise, if participants adopt a counting strategy whereby they increase their allocation of attention with each time marker, the precision of attentional allocation to the time of target onset should decrease with increasing variability, but not with temporal structure, resulting in the same pattern of means as that predicted by a statistical moment time code.

Temporal structure hypothesis. If temporal structure, rather than a set of statistical moments, is used to allocate attention to points in time, CRT should be shorter after rhythmic sequences than after randomly timed sequences, regardless of overall variability in the sequences.

\section{Method}

Participants. Ten undergraduate students (6 females, 4 lefthanded) at a large research university participated in this study for class credit. Ages ranged from 20 to 47 years, with a mean of 27 years. All reported normal or corrected vision.

Apparatus. All the stimuli and equipment were identical to those in Experiment 1.

Design. Figure 4 illustrates each type of condition. The design was a 2 (variability) $\times 2$ (temporal structure) repeated measures factorial, with two hanging cells that had no comparison across the temporal structure dimension: a condition in which the target appeared after a random ITI that we term the uncued CRT condition and a condition in which the target appeared after an isochronous series of four WSs that we term the isochronous condition.

Procedure. The testing procedure was identical to that in Experiment 1 . All the trial types were presented in random order to the participants. The participants were presented with targets that ap-

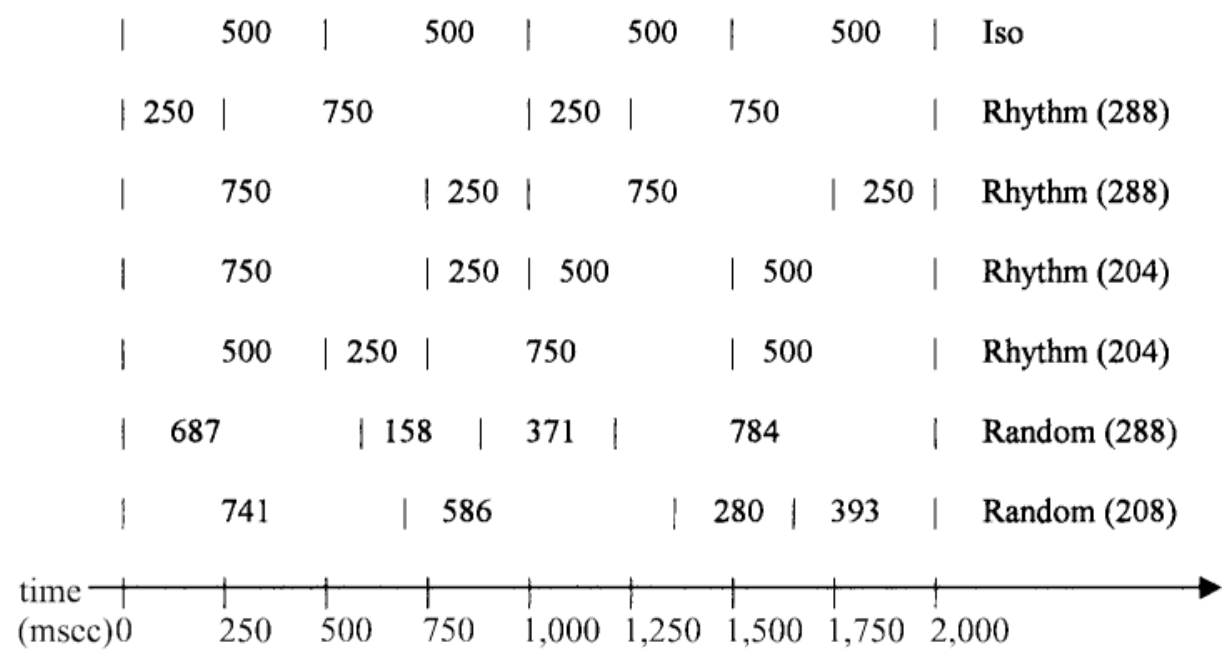

Figure 4. Illustration of the conditions used in Experiment 3. Numbers between hash marks represent stimulus interonset intervals (IOIs). Not all random sequences are shown: Six additional random sequences (three at each level of standard deviation) were created by randomly mixing the same IOIs as those illustrated above in the random conditions. 
Table 3

Mean Differences for Comparisons in Experiment 3

\begin{tabular}{lccrr}
\hline & Mean Difference & \multicolumn{1}{c}{$S E$} & U95\%CI & L95\%CI \\
\hline Structure & -24.781 & 3.424 & -17.033 & -32.529 \\
Variability & 6.903 & 3.615 & 15.083 & -1.277 \\
Iso: rhythmic & 17.775 & 8.693 & 37.445 & -1.895 \\
& -9.241 & 6.683 & 5.881 & -24.363 \\
Iso: random & 40.218 & 9.437 & 61.571 & 18.865 \\
& -4.565 & 6.033 & 9.086 & -18.216 \\
\hline
\end{tabular}

Note-SE, standard error of the mean; U95\%CI, upper $95 \%$ confidence interval; L95\% CI, lower 95\% confidence interval; Iso, isochronous.

peared after a random ITI (CRT trials) or after four WSs with IOIs arranged in one of five categorical structures: isochronous, rhythmic with a 204-msec standard deviation, rhythmic with a $288-\mathrm{msec}$ standard deviation, random with a 204-msec standard deviation, or random with a $288-\mathrm{msec}$ standard deviation. In all cases, the mean IOI was held constant at $500 \mathrm{msec}$. (See Figure 4 for the exact times and arrangements of IOIs used.) The rhythmic sequences were common musical rhythms made by arranging IOIs of 250, 500, and $750 \mathrm{msec}$, and two rhythms were created for each level of variance, for a total of four rhythms. Random IOIs were generated by selecting two sets of three digits from a random number table and then calculating the remaining two IOIs, subject to the constraint that the mean of the series was $500 \mathrm{msec}$ and the standard deviation was either 204 or $288 \mathrm{msec}$ (the standard deviation of the rhythmic sequences). Four random sequences were created for each level of variability by simply changing the order of IOIs, for a total of eight random sequences of IOIs. Only four random sequences (two at each level of variance) were seen by any single observer, in order to equate the repetition of specific sequences between the rhythmic and the random conditions. Two sets of four random sequences were used and entered into the analysis as a between-subjects variable. Each participant was observed 24 times in each condition.

\section{Results}

As in Experiments 1 and 2, the data were processed by first removing error trials, calculating a grand mean and standard deviation for each observer, and removing observations that fell outside of three standard deviations of the mean. This resulted in the loss of less than $10 \%$ of the data in all cases.

To probe for any effect or interaction of the specific set of random sequences seen by the observers, a 2 (standard deviation) $\times 2$ (random sequence version) mixed ANOVA was calculated. No effect of the specific set of random sequences was detected $[F(1,8)=1.775, p=$ $.219]$, nor was there an interaction between level of variability and specific sequence $[F(1,8)=0.156]$. In addition, each of the analyses below was performed with the sequence set variable as a between-subjects factor, and in none of the analyses did it have an effect or an interaction. Therefore, reported analyses are collapsed across this variable.

The means for each condition are presented in Figure 5. As in Experiments 1 and 2, a pseudo-omnibus $2 \times$ 2 repeated measures ANOVA comparing the variables of temporal structure (rhythmic vs. random) and standard deviation (204 vs. $288 \mathrm{msec}$ ) was performed. This test excluded the CRT and isochronous series conditions. This analysis revealed a main effect of temporal structure $[F(1,9)=52.387, p<.0005]$. The observers were quicker to respond to targets after rhythmic than after random sequences. There was no main effect of standard deviation $[F(1,9)=3.647, p=.088]$ and no interaction $[F(1,9)=0.199]$. Two ANOVAs served as planned com-

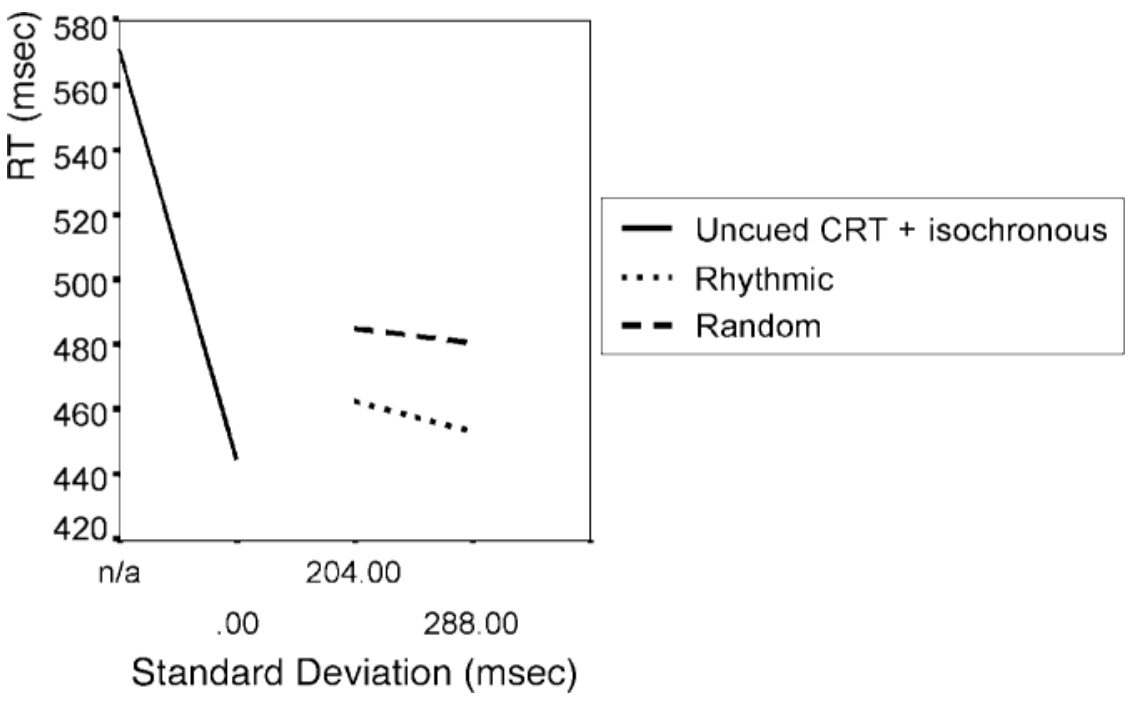

Figure 5. Mean reaction time (RT) for each condition in Experiment 3 as a function of standard deviation of warning stimulus sequence (which is not applicable to the uncued choice RT [CRT] condition). The solid line indicates uncued CRT and CRT following the isochronous series, the dotted line indicates the rhythmic series, and the dashed line indicates the random series. 
parisons, with Bonferroni adjusted $\alpha=.05 / 2=.025$. Mean differences for these analyses are presented in Table 3. The first analysis compared isochronous sequences with random sequences and revealed a main effect of variability [Hotelling's trace $F(2,8)=8.076, p=$ $.012]$. CRT was longer after random than after isochronous sequences. The second compared isochronous with rhythmic sequences and showed no main effect of rhythm [Hotelling's trace $F(2,8)=2.085, p=.187$ ], with a much smaller trend toward a longer CRT after rhythmic than after isochronous sequences.

Also, from the graph of means, it is clear that uncued CRT is longer than CRT to targets following random sequences. Post hoc analyses with Scheffé-corrected $S^{2}=$ 25.6 revealed that this difference was indeed significant for both a 204-msec standard deviation $[F(1,9)=44.8$, $p<.05]$ and a 288 -msec standard deviation $[F(1,9)=$ $34.9, p<.05]$.

Again, the analysis of proportion of correct responses failed to detect any effect of condition. The results of the logistic regressions for individual observers did detect a significant effect of trial type for 3 participants. For all 3 , the coded predictor indicating the uncued CRT condition (target after a random ITI) was significant and negative (with errors coded 0 , correct responses coded 1), indicating that the likelihood of an error was greater in the uncued CRT condition than would be predicted on the basis of chance alone.

\section{Discussion}

The main effect of temporal structure demonstrates that the pattern, and not merely the variability, of IOIs in a sequence determines the allocation of attention. This replicates the finding of Jones and Yee (1997) that detectability of temporal change is greater for rhythmic than for random sequences but extends it to CRT, a measure of efficiency, rather than sensitivity. This finding is more consistent with a coupled oscillator account of attentional allocation than with a stochastic timing mechanism or counting strategy. Although it is likely that a model could be conceived that would use information from stochastic timers to extract information about pattern, much as Povel's (1981) grid model does, such an account would again require that the relationship between attention and the time code be specified.

Again, there was no indication of a speed-accuracy tradeoff. To the contrary, the significant logistic regression for 3 participants indicates that there was a greater likelihood of error in the uncued CRT condition, which had the longest CRTs.

The results also address another issue concerning temporal attention. Although it is unlikely that any theory would predict better performance after random than after isochronous sequences, from a methodological standpoint it is possible that the number of WSs, rather than their temporal structure, causes the benefit for performance, relative to uncued CRT. Our post hoc analysis did indeed reveal a significant benefit for CRT after random sequences, relative to uncued CRT. It is not surprising that even random sequences would cause a benefit, since they would act as warnings of an impending target, if nothing else. However, isochronous sequences caused significantly shorter CRTs than random sequences did, and the difference between rhythmic and random sequences leaves little doubt that temporal structure matters, not just the number of WSs.

\section{EXPERIMENT 4}

We attributed the benefit to CRT observed in Experiment 1 to an allocation of attention in time, because there was no detectable speed-accuracy tradeoff and random target identity precluded motor preparation. The results of Experiment 3 indicate that attention depends on some mechanism that takes into account information other than statistical moments of durations. However, the evidence is not strong enough to rule out stochastic timing mechanisms as determinants of temporal attention.

Because a coupled oscillator necessarily mimics the statistical properties of the driver to which it is coupled, there is a seemingly unavoidable qualitative similarity between the predictions derived from coupled oscillator and stochastic timing accounts. Therefore, we took a different approach in Experiment 4. We used formalizations of the assertions of these theories to predict singletrial CRT data on an individual observer basis. This allowed us to compare quantitative, rather than qualitative, predictions of the theories. Such an approach has many pitfalls and, by itself, is unlikely to be definitive. However, a formal approach does complement more traditional qualitative studies.

Experiment 4 was a variation on a CRT experiment in which all the stimuli were targets occurring at some IOI after the previous target. A pulse was defined by the onset time of the first target and the most common IOI of $1,600 \mathrm{msec}$ (i.e., a rate of $0.625 \mathrm{~Hz}$ ). This stimulus pulse was then maintained, except for rare deviations. A minority of targets occurred at some lead with respect to the pulse, where lead is defined as the difference between the time point $1,600 \mathrm{msec}$ after the previous target and the time of target onset. An early target was followed by a target with an IOI that was extended by an amount equal to the early deviation, in order to reestablish the stimulus pulse. No late deviations were used in this design, because the absence of an expected event causes an orienting response (Sokolov, 1975), which would be an interesting phenomenon to investigate from a coupled oscillator perspective but is beyond the scope of the present article. The rate used was near the lower limit of behavioral synchronization (Fraisse, 1982) but had the advantage of allowing large perturbations of stimulus timing. Theoretically, large perturbations should correspond to a large effect size.

The general approach to modeling was to take stimulus onset times for an individual participant as input to a set of equations representing a particular cognitive model 
and to generate, for each stimulus and observer, a predicted level of attention. This was done for three cognitive models, including the formalization of DAT presented by Large and Jones (1999) and two temporal interval averaging processes, one with a long memory and one with a short memory. The predicted levels of attention derived from each of the cognitive models were then used as simultaneous predictors in an autoregressive integrated moving average (ARIMA) transfer function (Box \& Jenkins, 1970; see Gregson, 1983, for a treatment of ARIMA applications to psychology). The relationship between target identity and the identity of the previous two targets was also included in each participant's ARIMA transfer function, as will be described below. Two transfer functions were estimated for each participant, reflecting two assumptions about the relationship between attention and CRT. The first assumption is that CRT is simply negatively related to attention, so that as attention increases, CRT decreases linearly. Under this assumption, predicted attention levels should be negatively correlated with CRT. The second assumption is that CRT is an inverse function of attention. Under this assumption, attention should be positively correlated with the inverse of CRT, or response speed.

To derive predicted attention levels from DAT, we use the formalization of Large and Jones (1999; see also Batschelet, 1981). Attention in this model is represented as the von Mises circular probability distribution. Formally, the model is

$$
\begin{aligned}
& t_{n}=f(t) \\
& F\left(\phi_{n}\right)=\left(\frac{1}{2 \pi}\right) \sin \left(2 \pi \phi_{n}\right) \\
& \phi_{n+1}=\phi_{n}+\frac{t_{n+1}-t_{n}}{p_{n}}-\eta_{\phi} F\left(\phi_{n}\right) \bmod _{-.5,5} 1 \\
& p_{n+1}=p_{n}+p_{n} \eta_{p} F\left(\phi_{n}\right) \\
& s_{n+1}=s_{n}-\eta_{s}\left[s_{n}-\cos \left(2 \pi \phi_{n}\right)\right] \\
& \kappa_{n+1}=A^{-1}\left[\min \left(b, s_{n+1}\right)\right] \\
& \text { and } \\
& v_{n+1}=\frac{1}{I_{0}\left(\kappa_{n}\right)} \exp \left(\kappa_{n} \cos 2 \pi \phi_{n+1}\right),
\end{aligned}
$$

where $t_{n}$ is the onset time of stimulus $n, f(t)$ is the vector of stimulus onset times, $\phi_{n}$ is the phase of the attending rhythm at time $t_{n}, p$ is the period of the oscillator, $s$ is the synchronization strength (an index of the degree of correlation between the attending rhythm and the external event stream), $\kappa$ is a function of $s$ with an upper limit $b$ that represents concentrated attentional energy, the function $A^{-1}$ is found by table (see Batschelet, 1981), $\eta_{x}$ is a constant, and $v$ is the value of the von Mises distribution given $\kappa$ updated by the previous trial and the current phase of the attending rhythm. The value of $v$ is the predicted level of attention.

For the purpose of fitting the model to CRT, the phase was initially set to 0 at the onset of the third WS (i.e., the first stimulus onset time predictable from the previous
WS) and, thereafter, was allowed to vary iteratively. The period was likewise initially set to the period defined by the most common IOI, but iteration was not begun until the third stimulus, by which time the adapted period should be quite close to this most common period. Although these values represent imperfect assumptions, their exact values at the beginning do not greatly influence later values, since this system does not exhibit sensitive dependence on initial conditions with the parameters used here. The model has a limit cycle attractor in its state space, with a basin of attraction that covers the state space (i.e., the attractor is globally stable). Therefore, small errors in initial estimates will not have large consequences on later iteration values. The constant $\eta_{\phi}$ was set to 1, since the results of Experiment 1 and pilot work indicate that phase correction takes place very rapidly, as evidenced by the benefit after just two WSs (one interval). The constant $\eta_{p}$ was set to 0 , because only phasic period changes were present in the stimulus series, and $\eta_{s}$ was set to .25 , allowing the synchronization strength to slowly adapt to changes in the stimulus series. The maximum value of $s$ was set to .95 , corresponding to a $\kappa$ of 10.27 .

Chronometric predictions derived from timing theories depend on the relationship asserted between attention and the time code. Unfortunately, interval-timing theories that do specify a relationship between temporal information processing and attention tend to cast attention as cause, rather than as effect (e.g., Allan \& Kristofferson, 1974; Boltz, 1991; Brown, 1997; Lejeune, 1998, 2000; Macar, 2002; Macar, Grondin, \& Casini, 1994; Thomas \& Weaver, 1975). Several theories of alerting and RT assume that time information allows the focusing of attention, but these theories tend to neglect the timing aspect of performance and do not specify the form of the relationship beyond a more is better level.

Many such relationships are possible, and chronometric predictions depend heavily on which relationship is asserted. For example, the onset time of the attention window, the width of the window, the duration of the memory of both the attention and the time encoding processes, the nature of attentional updating based on time code updates, and perhaps other issues would have to be specified in order to make a comparison between interval-timing theories and DAT, which already specifies the relationship between stimulus onset times and attention. Exploring each of these issues is well beyond the scope of this article. In what follows, we do attempt a step in this direction by formalizing two potential relationships between a time code and attention.

We take the multiple-look model (Drake \& Botte, 1993) as our starting point. According to this model, the mean and variability of a sequence (modeled here as the arithmetic mean and standard deviation) are represented with some error that approaches some asymptotic value as the number of observed IOIs (i.e., sample size) approaches infinity. We assume here that this error is normally distributed about the true arithmetic mean duration of a series of IOIs, with a standard deviation that is determined 
by the standard deviation of the IOIs. Attention is then modeled as a normal distribution, with a maximum at the expected point defined as the onset time of the previous stimulus plus the mean IOI. This model has several desirable properties in common with DAT-particularly, that the intensity of the attentional pulse increases with increasing sample size and the width of the attentional window defined by the normal distribution decreases with decreasing variability of the input.

Formally, this model can be represented as follows:

$$
\begin{aligned}
& t_{n}=f(t) \\
& I_{n}=t_{n}-t_{n-1} \quad(n>1),\{\varnothing\} \text { otherwise } \\
& M_{n}=\frac{1}{n} \sum_{i=2}^{n} I O I_{i} \\
& s_{n}=\frac{1}{n} \sqrt{\sum_{i=3}^{n}\left(I O I_{i}-M_{i}\right)^{2}} \\
& a_{n}=\frac{n}{(\sqrt{2 \pi}) s_{n-1}} \exp \left(\frac{-\left[t_{n}-\left(t_{n-1}+M_{n-1}\right)\right]^{2}}{2 s_{n-1}^{2}}\right),
\end{aligned}
$$

where $t_{n}$ is the time of onset of stimulus $n, f(t)$ is the vector of stimulus onset times, $a_{n}$ is the level of attention at $t_{n}, I O I$ is the IOI between the current stimulus and the previous stimulus, $M$ is the arithmetic mean of all previous IOIs, and $s$ is the standard deviation of all previous IOIs. Note that $I O I$ and $M$ cannot be calculated until the second stimulus. Likewise, $s$ is undefined until the third stimulus onset, and $a$ is based on the immediately previous mean and standard deviation, so the model can predict CRT beginning with the fourth observation. For the remainder of this report, this model is referred to as the running average model, to distinguish it from the next model.

Finally, we also fit a moving average model, much like the running average model, but using only the previous three interval durations to calculate the means and standard deviations of IOIs. Conceptually, this model represents a process that has a short memory.

Each of these models makes similar qualitative predictions. Attention is predicted to increase with each repetition of the modal IOI (up to a limit for DAT). This is because of phase correction for DAT, and because of better estimation of the mean IOI for the average models. Each type of model predicts a lower level of attention to early targets with an increasing number of previous modal IOIs, because the attentional pulse becomes narrower with more presentations of the modal IOI, reflecting greater certainty about target onset time. Each model also predicts that attention will be reduced following an early onset, because of a sudden change in the time code (the phase of the attending rhythm in the case of DAT, the mean IOI in the case of the average models). Although the DAT model has four parameters (three weighting parameters and a limiting parameter) and the averaging models have none, all the parameters were set a priori for the DAT model and were not allowed to vary.
In addition to the formal models of attention, it may be expected that sequential effects of the stimulus category in a choice task will account for a significant portion of variance in CRT (Rabbitt \& Vyas, 1979). Specifically, if the previous stimulus is identical to the current stimulus, processing is facilitated relative to trials on which the current and the previous stimuli do not have the same identity. Consequently, although such sequential effects were not of primary interest, sequential match variables were created for lag 1 (i.e., the color on trials $n$ and $n-1$ were the same or different) and lag 2 (i.e., the color on trials $n$ and $n-2$ were the same or different), dummy coded as 0 for no match and 1 for a match.

The ARIMA transfer functions act as hierarchical regression models for an individual participant's time series, with autoregressive and moving average components entered first, followed by predictors that are external to the modeled time series. These external predictors are entered as a group, so only variance in the time series that covaries uniquely with a given predictor is used to determine the significance of that predictor. At $\alpha=.05$, one would expect 1 out of 20 parameter estimates for these external predictors to be significant by chance. In other words, if none of the attention models used here relate to CRT, we would still expect some of the transfer function parameters to be statistically significant. To address this issue, we calculated the binomial probability of the obtained number of significant fits, given an expected value of 0.4 significant fits and 7.6 insignificant fits (i.e., $p=$ $.05, q=.95$ ) to determine whether the observed frequency of significant prediction was greater than would be expected on the basis of chance.

\section{Method}

Participants. Eight undergraduate psychology students (7 females, 1 left-handed) at a large research university participated for extra credit in a psychology class. Ages ranged from 18 to 21 years, with a mean of 19.9 years. All reported normal or corrected vision.

Stimuli and Apparatus. All the equipment was identical to that in Experiment 1. Stimulus presentation was programmed using Presentation .46. Stimuli consisted of the letters $\mathrm{X}$ and $\mathrm{O}$, white against a black background, presented foveally for a duration of $34 \mathrm{msec}$ and subtending approximately $1^{\circ}$ of visual angle.

Design. The design can be conceived as a repeated measures factorial design with IOI as the single factor. It should be noted, however, that cell means were not analyzed with a repeated measures ANOVA but, rather, the time series of each observer's RTs were analyzed with an ARIMA transfer function.

Procedure. The participants were informed of the experimental procedure and of their rights as participants, and informed consent was obtained. The participants were told that they would see Xs and Os appear on the screen and that their task was to press the right mouse button if they saw an X and the left if they saw an O. Both speed and accuracy were emphasized. The participants were given no other information about the experiment prior to debriefing. Upon completion of the experiment, the participants were debriefed and given proof of attendance.

Seventy percent of the IOIs were $1600 \mathrm{msec}, 15 \%$ were one of eight shorter IOIs $(1,500,1,400,1,300,1,200,1,100,1,000,900$, or $800 \mathrm{msec}$ ), and the remaining $15 \%$ immediately followed a shorter IOI and compensated by an increase in duration equal to the previ- 


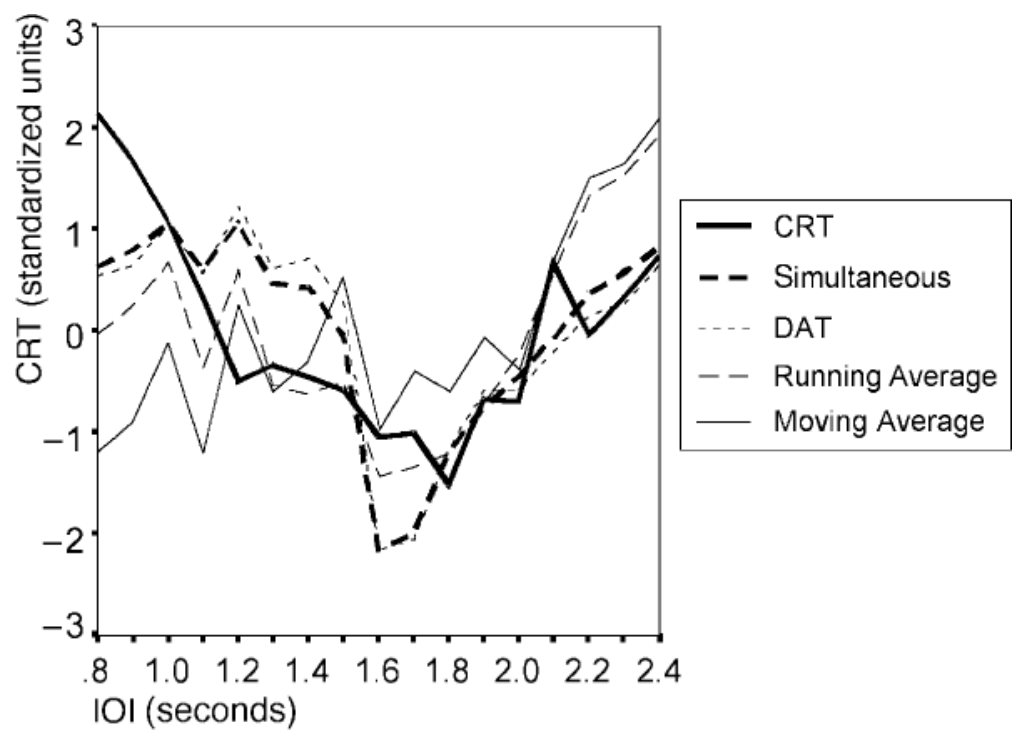

Figure 6. Observed and predicted means in Experiment 4, collapsed across participants. Units are standardized units. IOI is the interonset interval separating the target from the previous target. The bold solid line represents observed means. The heavy dashed line represents the mean predicted choice reaction time (CRT) of the simultaneous transfer function, which included theoretical attention levels from dynamic attending theory (DAT), the running average and moving average models as simultaneous predictor variables, in addition to autoregressive and lagged matching parameters. Parameter estimates for this transfer function are presented in Table 4. Other lines represent predictions for which only the indicated cognitive model's predicted attention levels were used, along with the autoregressive parameters and lagged matching variables.

ous decrease, in order to reestablish the pulse defined by the majority of IOIs. All the IOIs were presented in random order, subject to the constraint that an early onset could not occur after fewer than two 1,600-msec IOIs. Trials consisted of the presentation of a single target. Each participant was observed for a total of 1,060 trials. Sessions lasted approximately $30 \mathrm{~min}$.

\section{Results}

Error trials were treated as missing data in each CRT time series. The CRTs were nonstationary for all the participants, with a significant increasing trend with time. This trend was removed by regressing CRT on time of stimulus onset. We then identified $\operatorname{ARIMA}(p, d, q),(s p$, $s d, s q)^{3}$ order from the autocorrelation function and partial autocorrelation function of the residuals and estimated filters to account for autocorrelation. Once the model order was identified for an individual participant, an ARIMA transfer function was fit, using the lag 1 and lag 2 matching variables and the predicted attention levels from each of the three cognitive models as simultaneous predictors. Under the assumption of a linear attention-CRT relationship, the predicted attention levels were fit to the detrended CRT series. Under the assumption of an inverse attention-CRT relationship, the CRT series was inverted to produce response speed and then was detrended to produce the criterion variable.
Grand means for each IOI, averaged over participants, are shown in Figure 6 in standardized units. Also shown in the graph are mean CRTs (in standardized units) predicted by the simultaneous transfer functions, collapsed across participants, assuming a linear attention-CRT relationship. Finally, to illustrate predictions derived from each cognitive model by itself, Figure 6 includes predicted mean CRTs from transfer functions that included the same autoregressive moving average and lagged matching predictors, plus the single cognitive model prediction indicated. From the graph, it is evident that each cognitive model underestimates CRT following short IOIs and that the statistical models overestimate CRT following long IOIs.

Estimates of the transfer function slopes are presented for each participant and cognitive model in Table 4. Only the predictors of interest are included in the table. Each participant's ARIMA also included autoregressive parameters indicated by the autocorrelation function and partial autocorrelation function, the order of which was unique to each observer. A statistically significant slope is interpreted just as a regression coefficient is interpreted but applies only to a single participant. For example, for Participant 7, both the attention level predicted by DAT and that predicted by the running average model were significant simultaneous predictors of CRT. 
Table 4

\begin{tabular}{|c|c|c|c|c|}
\hline \multicolumn{5}{|c|}{$\begin{array}{l}\text { Slopes of Transfer Functions Relating Cognitive Model Predictions to Choice } \\
\text { Reaction Time (CRT) }\end{array}$} \\
\hline Observer & Relationship & DAT & Running Average & Moving Average \\
\hline \multirow[t]{2}{*}{1} & CRT & -2.84 & -1.85 & -0.01 \\
\hline & Response speed & $1.619 \mathrm{E}-05^{*}$ & 8.42E-06 & 4.00E-08 \\
\hline \multirow[t]{2}{*}{2} & CRT & $-3.40 *$ & -6.78 & 0.00 \\
\hline & Response speed & $1.81 \mathrm{E}-05^{*}$ & 3.71E-05 & $2.00 \mathrm{E}-08$ \\
\hline \multirow[t]{2}{*}{3} & CRT & 3.40 & -2.75 & 0.01 \\
\hline & Response speed & $-3.96 \mathrm{E}-05$ & $2.35 \mathrm{E}-05$ & $-2.00 \mathrm{E}-08$ \\
\hline \multirow[t]{2}{*}{4} & CRT & $-8.28 * *$ & -136.5 & 0.00 \\
\hline & Response speed & 2.42E-05 & 2.02E-04 & 7.00E-08 \\
\hline \multirow[t]{2}{*}{5} & CRT & -0.04 & -13.91 & -0.02 \\
\hline & Response speed & $-6.95 \mathrm{E}-05$ & $-1.39 \mathrm{E}-04$ & $1.50 \mathrm{E}-07$ \\
\hline \multirow[t]{2}{*}{6} & CRT & $-2.72 *$ & -8.30 & 0.00 \\
\hline & Response speed & $2.15 \mathrm{E}-05^{*}$ & 6.32E-05 & $-4.00 \mathrm{E}-08$ \\
\hline \multirow[t]{2}{*}{7} & CRT & $-4.59 * *$ & $-13.53^{* *}$ & 0.00 \\
\hline & Response speed & $2.97 \mathrm{E}-05^{*}$ & $9.87 \mathrm{E}-05^{* *}$ & $-1.00 \mathrm{E}-08$ \\
\hline \multirow[t]{2}{*}{8} & CRT & $-5.30 * *$ & 0.39 & 0.00 \\
\hline & Response speed & $3.40 \mathrm{E}-05^{* *}$ & $-3.85 \mathrm{E}-06$ & $1.00 \mathrm{E}-08$ \\
\hline
\end{tabular}

Note-Each predictor variable is a time series of predicted attention levels derived from equations presented in the text. Significance levels represent only variance that is uniquely accounted for by a given predictor when all other predictors and ARIMA parameters are included. Included in each ARIMA, but not in the table, were autoregressive and moving average parameters (the order of which was unique to each participant) and the lagged sequential matching variables described in the text.

$* p<.05$. ** $p<.005$.

The binomial probability, $p\left(x=f ; N, p_{s}\right)$, where $x$ is the number of "successes," or significant fits, $p_{s}=$ probability of significant fit $=.05$ and $q=.95$, and $f$ is the observed frequency of significant fits, was calculated for $N=8$. The predicted attention values derived from DAT accounted for unique variance in CRT for 6 observers. This is more than would be expected by chance $(f=6$, $p<.0001)$. DAT also accounted for response speed in 5 observers $(f=5, p<.0001)$. The running average model predicted CRT and response speed for only a single participant, which is not significantly different from what would be expected by chance. The moving average model was not a significant predictor of CRT or response speed for any observer.

Residuals of the ARIMAs were examined for remaining autocorrelation up to lag 20, and none was found.

Errors were analyzed with binary logistic regression, using the same predictors of attention level and lagged target repetitions. Since error rates were very low (proportion of errors ranged from .11 to .02; mean, .05), none of the variables were significantly able to improve the predicted categorization above predicting all correct responses (i.e., a model with only a constant).

\section{Discussion}

The purpose of Experiment 4 was to test alternative formal models consistent with either a coupled oscillator mechanism or a statistical information processing mechanism of temporally guided attention. In general, the statistical models were not good predictors of CRT. The running average model was a significant predictor of both response latency and response speed for only a single participant, whereas the moving average model was not significantly related to CRT or speed for any observer. In contrast, the DAT model was a significant predictor of CRT and speed for more observers than would be expected by chance. This result supports the idea that attention can be guided to points in time by a coupled oscillator mechanism.

This result does not rule out stochastic timing mechanisms as possible determinants of attention. Symbolic information from a single cue about when a target will occur has also been shown to cause a benefit to CRT (Kingstone, 1992), and in this case, entrainment is not possible, because there is nothing to drive an attending rhythm. Also, only two possible relationships between attention and CRT were tested here, but the question of the relationship between attention and CRT has not been definitively decided, as indeed the definition of attention remains controversial (Kinchla, 1992; Pashler, 1998, p. 1). Under different assumptions, stochastic models may be better predictors of CRT. However, the assumptions used here are reasonable and are the most common in current attention research (cf. Pashler, 1998, pp. 167-170).

\section{GENERAL DISCUSSION}

Experiment 1 conceptually replicated previous findings that temporal structure could act to facilitate the processing of stimuli. The unique features of this experiment were control of elapsed time and the extension of such findings to CRT along an uncued dimension without a speed-accuracy tradeoff. The results implicate guided attention in addition to increasing allocation of attention caused by changes in conditional probability of a target onset with elapsed time. 
In Experiment 2, temporal limits to guided attention were explored. At IOIs between 4 and $16 \mathrm{sec}$, ITIs between 12 and $14 \mathrm{sec}$, and a sequence presentation rate of $0.25 \mathrm{~Hz}$, no benefit of temporal structure was detectable, nor was an effect of elapsed time detected. This result is consistent with that of Requin and Granjon (1969), who used IOIs between 8 and $28 \mathrm{sec}$ and failed to detect a benefit to simple RT with elapsed time. The discrepancy between the results of Experiment 1 and those in Requin and Granjon may be in part due to the difference in time scales between the two experiments. This result indicates that models of temporal attention could benefit from explicit recognition of the time scale (i.e., rate of presentation). Although it might be the case that different mechanisms mediate timing in the milliseconds and seconds to minutes ranges, it would be beneficial to understand why this is the case. In the attention domain, what is the relationship between dynamic attending and vigilance, and where does the distinction between the two occur?

Experiment 3 demonstrated that temporal structure affects information processing efficiency, even when variability is controlled. Such a structure is readily encoded by coupled oscillator systems but cannot be encoded by a mechanism that uses only the moments of IOIs. Beatbased models (i.e., Povel, 1981) might also explain the results if the relationship between the time code and attention were specified, but to our knowledge, this has not been done.

Experiment 4 demonstrated that under the two common assumptions of a linear relationship between attention and response latency or response speed, a coupled oscillator model was a better predictor of sequential CRT than stochastic timing models were. This does not refute the assertion that a stochastic timing mechanism may be used to guide attention to points in time under certain circumstances but does provide support for the assertion that a coupled oscillator does guide attention in the presence of temporal structure. An interesting challenge for future research is to characterize the relationship between temporally guided attention in the presence of temporal structure and in the presence of symbolic cues, where entrainment is not possible.

In light of previous evidence for entrained attention, this set of experiments provides converging evidence from two approaches (qualitative and quantitative) to chronometric data for the role of a coupled oscillator in mediating the effects of temporal structure on behavior. The development of coupled oscillator models holds a great deal of explanatory promise for temporal attention. There are many interesting ways in which these models may be developed. One neglected aspect thus far has been the nature of perceptual coupling. How is the strength of perceptual coupling between environment and endogenous rhythms altered over time? Might the strength of this coupling be a determinant of whether one employs a top-down or a bottom-up strategy for tracking temporal structure? Another issue is the adaptability of such rhythms. Do attending rhythms have a preferred frequency, and what are the temporal limits of entrainment? Are there several internal oscillators with different frequencies? What is the relationship between attending rhythms and prominent neuronal population oscillations that have been observed in humans at theta-, alpha-, and gamma-band frequencies? These questions remain largely unexplored.

\section{REFERENCES}

Allan, L. G. (1979). The perception of time. Perception \& Psychophysics, 26, 340-354.

Allan, L. G., \& Kristofferson, A. B. (1974). Successiveness discrimination: Two models. Perception \& Psychophysics, 15, 37-46.

BARnes, R., \& Jones, M. R. (2000). Expectancy, attention and time. Cognitive Psychology, 41, 254-311.

Batschelet, E. (1981). Circular statistics in biology. London: Academic Press.

BoltZ, M. (1991). Time estimation and attentional perspective. Perception \& Psychophysics, 49, 422-433.

Box, G. E. P., \& JEnKIns, G.M. (1970). Time series analysis: Forecasting and control. San Francisco: Holden-Day.

BRown, S. W. (1997). Attentional resources in timing: Interference effects in concurrent temporal and nontemporal working memory tasks. Perception \& Psychophysics, 59, 1118-1140.

DraKe, C., \& BotTe, M.-C. (1993). Tempo sensitivity in auditory sequences: Evidence for a multiple-look model. Perception \& Psychophysics, 54, 277-286.

DunCAN, J. (1980). The demonstration of capacity limitation. Cognitive Psychology, 12, 75-96.

DunCAN, J. (1984). Selective attention and the organization of visual information. Journal of Experimental Psychology: General, 113, 501517.

Fraisse, P. (1982). Rhythm and tempo. In D. Deutsch (Ed.), The psychology of music (pp. 149-180). New York: Academic Press.

GARNER, W. R. (1985). Contingent information processing: Contingent and precued classification. Perception \& Psychophysics, 38, 237-248.

GARNER, W. R. (1987). Location and color as cuing dimensions in contingent classification. Perception \& Psychophysics, 41, 202-210.

GetTy, D. J. (1975). Discrimination of short temporal intervals: A comparison of two models. Perception \& Psychophysics, 18, 1-8.

Granjon, M., Requin, J., Durup, H., \& Reynard, G. (1973). Effects of a timing signal on simple reaction time with "non-aging" foreperiods. Journal of Experimental Psychology, 101, 139-145.

Gregson, R. A. M. (1983). Time series in psychology. Hillsdale, NJ: Erlbaum.

Grondin, S., Meilleur-Wells, G., \& Lachance, R. (1999). When to start explicit counting in a time-intervals discrimination task: A critical point in the timing process of humans. Journal of Experimental Psychology: Human Perception \& Performance, 25, 993-1004.

JoNES, M. R. (1976). Time, our lost dimension: Toward a new theory of perception, attention, and memory. Psychological Review, 83, 323-335.

JonEs, M. R., \& BolTZ, M. (1989). Dynamic attending and responses to time. Psychological Review, 96, 459-491.

Jones, M. R., \& YeE, W. (1997). Sensitivity to time change: The role of context and skill. Journal of Experimental Psychology: Human Perception \& Performance, 23, 693-709.

KilleEN, P. R., \& Weiss, N. A. (1987). Optimal timing and the Weber function. Psychological Review, 94, 455-468.

Kinchla, R. A. (1992). Attention. Annual Review of Psychology, 43, 711-742.

KINGSTONE, A. (1992). Combining expectancies. Quarterly Journal of Experimental Psychology, 44A, 69-104.

KLEMMER, E. T. (1956). Time uncertainty in simple reaction time. Journal of Experimental Psychology, 51, 179-184.

LARGE, E. W., \& JoNes, M. R. (1999). The dynamics of attending: How people track time-varying events. Psychological Review, 106, 119-159. 
LEJEUNE, H. (1998). Switching or gating? The attentional challenge in cognitive models of psychological time. Behavioural Processes, 44, 127-145.

LEJEUNE, H. (2000). Prospective timing, attention and the switch. A response to "Gating or switching? Gating is a better model of prospective timing" by Zakay. Behavioural Processes, 52, 71-76.

LuCE, R. D. (1984). Response times. New York: Oxford University Press.

MACAR, F. (2002). Expectancy, controlled attention and automatic attention in prospective temporal judgments. Acta Psychologica, 111, 243-262.

MACAR, F., Grondin, S., \& CASINI, L. (1994). Controlled attention sharing influences time estimation. Memory \& Cognition, 22, 673-686.

Maxwell, S. E., \& Delaney, H. D. (2003). Designing experiments and analyzing data: A model comparison perspective (2nd ed.). Mahwah, NJ: Erlbaum.

MCAuley, D. J., \& KIDD, G. R. (1998). Effect of deviations from temporal expectations on tempo discrimination of isochronous tone sequences. Journal of Experimental Psychology: Human Perception \& Performance, 24, 745-755.

Monahan, C. B., \& Hirsh, I. J. (1990). Studies in auditory timing: 2. Rhythm patterns. Perception \& Psychophysics, 47, 227-242.

Mowbray, G. H. (1964). Subjective expectancy and choice reaction times. Quarterly Journal of Experimental Psychology, 16, 216-223.

NÄÄTÄNEN, R. (1970). The diminishing time-uncertainty with the lapse of time after the warning signal in reaction-time experiments with varying fore-periods. Acta Psychologica, 34, 399-418.

NIEMI, P., \& NÄ̈̈TÄNEN, R. (1981). Foreperiod and simple reaction time. Psychological Bulletin, 89, 133-162.

Olsen, I. R., \& ChUN, M. M. (2001). Temporal contextual cuing of visual attention. Journal of Experimental Psychology: Learning, Memory, \& Cognition, 27, 1299-1313.

PaSHLER, H. E. (199) The psychology of attention. Cambridge, MA: MIT Press.

Poppel, E. (1997). A hierarchical model of temporal perception. Trends in Cognitive Sciences, 1, 56-61.

PoSNER, M. I. (1980). Orienting of attention. Quarterly Journal of Experimental Psychology, 32, 3-25.

PosNer, M. I., SNYDER, C. R., \& DAVIDSON, B. J. (1980). Attention and the detection of signals. Journal of Experimental Psychology: General, 109, 160-174.

PoVEL, J. D. (1981). Internal representation of simple temporal patterns. Journal of Experimental Psychology: Human Perception \& Performance, 7, 3-18.

Povel, J. D., \& Essens, P. J. (1985). Perception of temporal patterns. Musical Perception, 2, 411-440.

RABBITT, P. M. A., \& VYAS, S. M. (1979). Signal recency effects can be distinguished from signal repetition effects in serial CRT tasks. Canadian Journal of Psychology, 33, 88-95.
Requin, J., \& GRANJON, M. (1969). The effect of conditional probability of the response signal on simple reaction time. Acta Psychologica, 31, 129-144.

SiMON, J. R., \& Slaviero, D. P. (1975). Differential effects of a foreperiod countdown procedure on simple and choice reaction time. Journal of Motor Behavior, 7, 9-14.

SoKolov, E. N. (1975). The neural mechanisms of the orienting reflex. In E. N. Sokolov \& O. S. Vinogradova (Eds.), Neural mechanisms of the orienting reflex (pp. 217-235). Hillsdale, NJ: Erlbaum.

TeIchner, W. H. (1954). Recent studies of simple reaction time. Psychological Bulletin, 51, 128-149.

Thomas, E. A. C., \& WeAver, W. B. (1975). Cognitive processing and time perception. Perception \& Psychophysics, 17, 363-367.

TsaL, Y. (1983). On interpreting the effects of location preknowledge: A critique of Duncan. Perception \& Psychophysics, 34, 297-298.

Woodrow, H. (1914). The measurement of attention. Psychological Monographs, 17, 1-158.

Woodrow, H. (1930). The reproduction of temporal intervals. Journal of Experimental Psychology, 13, 473-499.

\section{NOTES}

1. Alternatively, one can use a Poisson distribution of foreperiods (and number of WSs) with respect to time, which makes the conditional probability of a target, given that no target has yet occurred, equal at all intervals (Luce, 1984, pp. 13-15; Mowbray, 1964). We prefer the former choice, since controlling the conditional probability of a target alters the simple probability of a target at each time point, in a direction opposite to that of the predicted effect. Also, in the context of time markers, altering the distribution of number of WSs does not solve the inherent confound of number of WSs with elapsed time.

2. Because the single-WS condition at the shortest IOI and the CRT condition do not have comparison conditions across the time marker dimension, the design is not crossed. Therefore, no true omnibus is possible that includes both CRT trials and trials with one WS and the shortest IOI.

3. Model order refers to the number of autoregressive, lagged difference, and moving average parameters that are necessary to account for the autocorrelational structure of the time series: $p=$ number of autoregressive parameters, $d=$ number of lagged differences necessary to make the time series stationary, $q=$ number of moving average parameters, $s=$ number of seasonal (cyclical) $p, d$, or $q$ parameters.

(Manuscript received May 5, 2003; revision accepted for publication April 27, 2004.) 